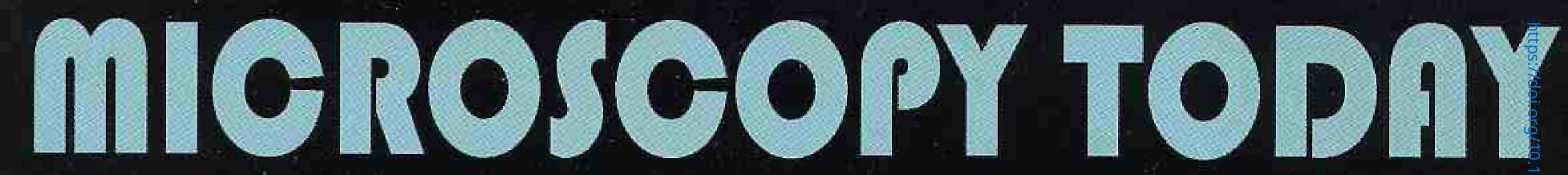
nOVEMBER/DEOEMIIBER 2003 VOLUME II AUMBER 6
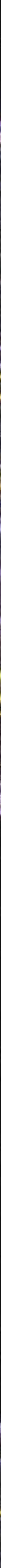


\section{Three NEW}

\section{DualBeams for}

Any Lab, Every
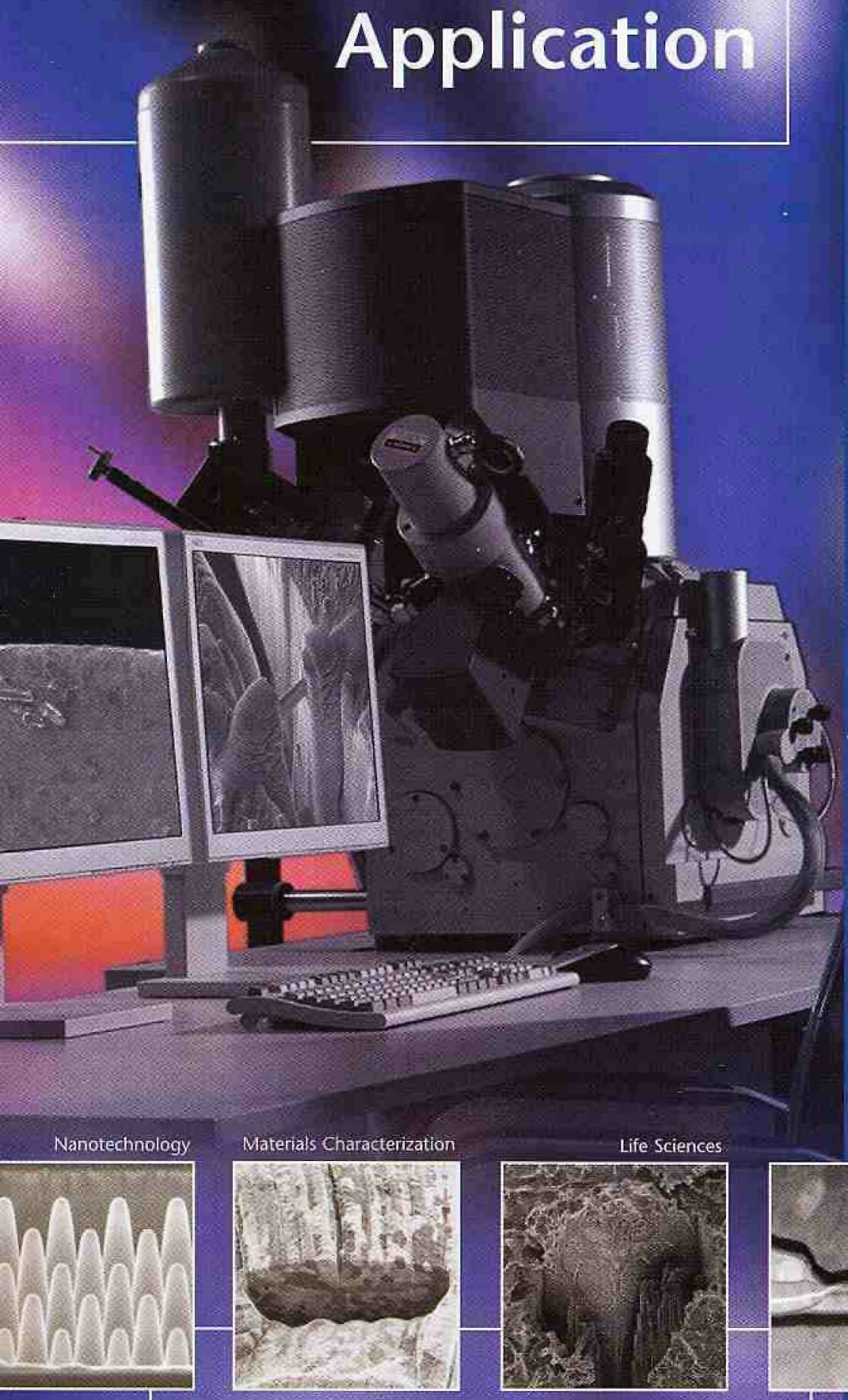

Optimized Solutions

Taking SEM Performance to a New Dimension
Now there is a DualBeam ${ }^{\text {M }}$ (FIB/SEM) for every lab, every application and every price/performance requirement. Introducing three fully-integrated and flexible DualBeam solutions featuring application-optimized operation, ensuring that your next system investment will meet your most specific needs.

NEW QuantaTM 3D - Combines the power of FIB for sample modification, high-vac, low-vac and ESEM performance, and 3D data reconstruction for advanced sub-surface materials characterization, diagnostics and process control.

New Nova'M NanoLab - A complete nanotechnology lab in a single tool, ideal for nanoscale machining, prototyping, characterization and analysis of complex structures below $100 \mathrm{~nm}$.

NEW Strata ${ }^{M}$ DB-STEM - Delivers integrated sample preparation, utira-high resolution STEM imaging and microanalysis below $1 \mathrm{~nm}$, for high throughput semiconductor and data storage labs.

Which DualBeam will you choose? For more information or a demonstration, contact your local sales representative.

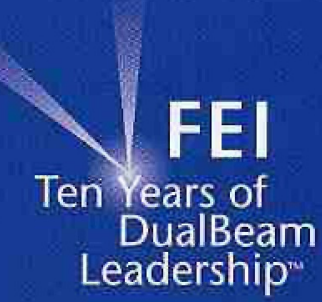

Semicondurtors Datastorage

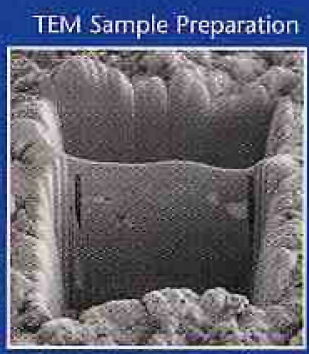

www.feicompany.com sales@feico.com 


\section{Sub-Ângstrom Resolution}

Stephen W. Carmichael

carmichael.stephen@mayo.edu

Antoni van Leeuwenhoek showed the practical use of the light microscope in the 1600 s after much effort to improve the quality of optical lenses. Pioneering microscopists such as Ernst Abbé, Hermann Ludwig Ferdinand von Helmholtz, Lord John Rayleigh, Carl Zeiss, and August Köhler then brought us to the brink of optimal performance of the light microscope approximately a century ago. Ernst Ruska and Max Knoll showed in the 1930s that high-energy electrons could be used in place of light, giving greatly improved resolution. In the 1970's Albert Crewe and co-workers developed the scanning transmission electron microscope (STEM) and used the Z-contrast method to improve resolution in the electron microscope by about a factor of two. The scanning probe (nonoptical) microscopes aside, there hasn't been a significant advance in spatial resolution since.

It has been well appreciated that spherical and chromatic aberrations have limited resolution in transmission electron microscopes (TEM) as well as limiting the probe size in the STEM. Philip Batson, Niklas Dellby, and Ondrej Krivanek have greatly modified a commercially available VG Microscopes STEM to achieve a probe size of $0.74 A$ and a consequent resolution of $0.76 \mathrm{~A}^{2}$, this is the best probe size ever reported for an electron microscope.

The microscope was modified to correct spherical aberration, and the STEM design was chosen since it is less sensitive to chromatic aberration. The heart of the aberration correction was the introduction of seven new sets of electromagnetic lenses (four quadrupoles separated by three octupoles, with extremely stable computer-controlled current supplies) into the optical pathway. Detectors (television cameras) were placed in the far field behind the specimen. The software then corrects the detected aberrations, by introducing distortions to oppose existing aberrations, canceling them out.

Batson et al. pointed out four recent technical advances that made these improvements possible. First, computation of electron optical parameters is now possible, allowing practical designs to be devised. Second, there have been significant advances in mechanical fabrication tolerances. Third, the stability of electronic components has improved recently, allowing for exquisite control of currents within electromagnetic lenses. And fourth, the computing capabilities to provide real-time feedback between the detectors and lenses are now readily available.

Using this improved instrument with resolving power below the diameter of a hydrogen atom, Batson et al. are using it to image single atom defects in inorganic specimens. The usefulness of this instrument in monitoring nanofabrication was obvious. The potential of employing such a STEM in a manner somewhat similar to confocal light microscopy to image subcellular structure at the atomic level in biologic specimens is also very tantalizing. Nevertheless, Batson et al. have made the most significant breakthrough in optical microscopy in decades. I can hardly wait to see how this new, improved design will be put to use!

\section{Footnotes}

${ }^{1}$ The author gratefully acknowledges Dr. Philip Batson for reviewing this article.

2 Batson, P.E., N. Dellby, and O.L. Krivanek, Sub-ängstrom resolution using aberration corrected electron optics, Nature 418:617-620, 2002.

\section{InDex of Antigtes}

The Scanning Confocal Electron Microscope...

Nestor J. Zaluzec, Argonne National Laboratory

Correlative Electron Tomography And Elemental Microanalysis

In Biology: A Preview

Michael Marko, Terence Wagenknecht, and Carmen Mannella Wadsworth Center

Creating Pseudocolored Images in Photoshop. Jerry Sedgewick, University of Mimesota

UItra Low Voltage BSE Imaging .. Michael D.G. Steigerwald, LEO Electron Microscopy

Techniques for Combinatorial and High-Throughput Microscopy

Part 2: Automated Optical Microscopy Platform for

Thin Film Research

S.-W. Kim, A. Sehgal, A. Karim, and M.J. Fasolka,

Polymers Division, National Institutes of Standards and Technology

Microscopy of Silicified Wood George Mustoe, Western Washington University

Microwave Processing in Diagnostic Electron Microscopy Ross G. Gerrity and George W. Forbes, Medical College of Georgia

A Polarizing Microscope for Mapping Birefringent

Objects in 3D Space Michael Shribak and Rudolf Oldenbourg, Marine Biological Laboratory

Colocalization of Fluorescent Probes

Using Image-Pro ${ }^{\mathbb{1}}$ Plus v. $\mathbf{5 . 0}$ Will Casavan and Yuri Gaidoukevitch, Media Cybernetics, Inc.

Electron Microscopy in Australia Paul Munroe, University of New South Wales

Converting Right-Left Stereo Pairs Into Colored Pairs

For Electronic Presentation

Mark W. Tengowski, Pfizer Inc.

Cellulose Acetate Replication of Plant Surfaces for SEM Gib Ahistrand, University of Minnesota

8
Safe Handling of Embedding Media

.54

E. Ann Ellis, Texas A \& M University

Industry News.

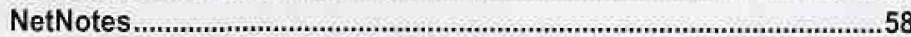

Microscopes as Gifts

Caroline Schooley

individuals to be Considered for

Major Awards by the Microscopy Society Of America

Microscopy Society of America Position on

Ethical Digital Imaging

Index of Advertisers.

(1)

\section{ce} cellei in green. This fluorescence micrograph was acquired in the laboratory of Dr. Clare Waterman-Storer at the Scripps Research Institute in La Jolla, CA, and won first place in the 2003 Nikon International Small World Photomicrography contest. 